\title{
Autonomía del trabajo y satisfacción laboral en trabajadores de una universidad peruana
}

\author{
${ }^{1}$ Adela Faya Salas, ${ }^{2}$ Carlos Venturo Orbegoso, ${ }^{3}$ Miriam Herrera Salazar y ${ }^{4}$ Ronald M. \\ Hernández ${ }^{\mathrm{a}}$ \\ ${ }^{1}$ Universidad César Vallejo, Lima, Perú. \\ ${ }^{1}$ Universidad Alas Peruanas, Lima, Perú \\ ${ }^{2}$ Universidad César Vallejo, Lima, Perú. \\ ${ }^{3}$ Universidad Autónoma del Perú, Lima, Perú \\ ${ }^{4}$ Universidad San Ignacio de Loyola, Lima, Perú. \\ ${ }^{1}$ ORCID ID: https://orcid.org/0000-0002-7991-4133 \\ ${ }^{2}$ ORCID ID: https://orcid.org/0000-0002-7465-8687 \\ ${ }^{3}$ ORCID ID: https://orcid.org/0000-0001-5249-6915 \\ ${ }^{4}$ ORCID ID: https://orcid.org/0000-0003-1263-2454
}

Recibido: 12 de febrero de 2018

Aceptado: 01 de julio de 2018

\section{Resumen}

La investigación tuvo como objetivo determinar la relación que existe entre la autonomía del trabajo y la satisfacción laboral de los trabajadores administrativos de una universidad peruana. Se empleó el método hipotético deductivo, basado en un enfoque cuantitativo de diseño no experimental y de corte transversal. Se aplicó un Cuestionario a 122 trabajadores. Los resultados permitieron determinar la correlación directa, moderada y significativa, con un coeficiente de correlación de Spearman Rho $=0.651^{* *}$ y $0.626^{* *}$ y una significancia $($ Bilateral $)=0.000(\mathrm{p}<.05)$. Por lo cual se llegó a la conclusión, que cuando la autonomía del trabajo es real, el nivel de satisfacción laboral es alto, en los trabajadores de ambos sexos.

Palabras clave: Autonomía del trabajo, Satisfacción laboral, personal administrativo, universidad.

\section{Autonomy of Work and Job Satisfaction in Administrative Workers}

\section{Abstract}

${ }^{\text {a }}$ Correspondencia al autor

E-mail: rhernandezv@usil.edu.pe 
The objective of the research was to determine the relationship between the autonomy of work and the job satisfaction of the administrative workers of a Peruvian university. The hypothetical deductive method was used, based on a non-experimental, cross-section quantitative design approach. A questionnaire was applied to 122 workers. The results allowed the researchers to determine the direct, moderate and significant correlation, with a Spearman correlation coefficient $\mathrm{Rho}=0.651 * *$ and $0.626 * *$ and a (Bilateral) significance $=0.000(\mathrm{p}<.05)$. Therefore, it was concluded that when work autonomy is $\mathrm{pr}$, the level of job satisfaction is high in workers of both sexes.

Keywords: Autonomy of work, Job satisfaction, administrative staff, university.

\section{Introducción}

En la actualidad, se observa que una de las mayores preocupaciones de los gestores empresariales radica en poder contar con trabajadores satisfechos en sus labores, motivados, productivos y comprometidos con el logro de metas y el desarrollo de las organizaciones (Moreno, et al., 2015; Mirás, Villoria, Álvarez, Cotobal, \& González, 2017). Diversos investigadores han concluido que las organizaciones que tienen clientes con altos niveles de satisfacción, también cuentan con trabajadores con altos niveles de satisfacción laboral. Por ende, se vienen estudiando y aplicando, en las organizaciones, diversas teorías que ayuden a incrementar significativamente la satisfacción laboral de todo el personal.

Herzberg (1959) desarrolló la teoría bifactorial, concluyendo que la insatisfacción laboral depende de factores denominados higiénicos como el ambiente, la supervisión, los colegas y el contexto general del cargo; y que la satisfacción laboral está en función del contenido o de las acciones desafiantes y estimulantes de la función, denominados factores motivadores. En ese sentido, propuso el enriquecimiento de las tareas para lograr la satisfacción en el trabajo, convirtiendo a éstas en desafíos a través del incremento de la responsabilidad y de los objetivos. Por su parte, Schein (1990) señaló que la autonomía e independencia son factores que caracterizan a las personas que buscan situaciones laborales en las que pueden sentirse libres de las limitaciones impuestas por la organización para desarrollar sus competencias, lo cual les permite ser más productivos.

En la actualidad; la competencia de las empresas, por obtener cada vez mayor participación de mercado y garantizar su supervivencia o liderar su sector productivo (Bonilla \& Bonilla, 2016), ha obligado a los empresarios a tener una mayor preocupación por sus trabajadores, que a la larga son los que con su productividad permitirán alcanzar los objetivos empresariales (Gómez \& Barboza, 2015). En este sentido; Luceño, Martí, Rubio y Jaén (2008) afirmaron: 
Gracias a la automatización de procesos, las nuevas tecnologías, la bioingeniería, la ergonomía, etc., la naturaleza del trabajo ha experimentado grandes cambios, desde tareas caracterizadas por altas demandas físicas a empleos cuyas principales exigencias son mentales y emocionales. Igualmente se han modificado las características de la masa laboral, destacando entre otros, la incorporación de la mujer al mercado de trabajo, un rápido envejecimiento de la población, la apertura de las fronteras entre los países, etc. (p. 116).

Para garantizar su competitividad y productividad, las organizaciones buscan reclutar a los mejores trabajadores y fidelizarlos en sus posiciones laborales. Esta es una tarea difícil que enfrentan las empresas de hoy, porque las nuevas generaciones tienen mejores expectativas que antes. De acuerdo con Arellano Marketing (2013), los jóvenes aprecian en mayor medida un estilo de vida fundamentalmente sofisticado, a los peruanos les agrada más empleos que "suenen interesantes", y mientras los más jóvenes buscan un aprendizaje continuo, los de mayor edad y grados académico valoran más los beneficios pecuniarios. Por su parte; Granada (2006), Solórzano (2018) afirmaron que la insatisfacción laboral como factor del bajo rendimiento del trabajador es importante, y se puede evidenciar que, en algunas instituciones, pese a sus grandes esfuerzos para ampliar y modernizar su equipamiento, afrontan problemas de rendimiento laboral. De acuerdo con Eurofound and EU-OSHA (2014), los trabajadores con más autonomía laboral sufren menos estrés que aquellos trabajadores con empleos igualmente demandantes, pero con menos autonomía (Miranda-Ledesma \& Batista-Anache, 2018)

En este contexto, la autonomía del trabajador puede ser un importante factor protector frente al estrés. La autonomía laboral podría mejorarse, aumentando la capacidad del empleado en influir en su ambiente laboral. Por ejemplo, otorgándole la capacidad de cambiar el orden de las tareas, el método de trabajo, la velocidad o el ritmo de trabajo, el calendario de sus descansos, o dándole voz y voto en la elección de determinadas decisiones de la empresa. De esta manera, en los puestos de trabajo que requieren alta demanda, tal y como se establece en el informe de la EU-OSHA y del Eurofound, la autonomía juega un papel determinante en la prevención del estrés, mejorando el rendimiento de los trabajadores y reduciendo el absentismo laboral.

Ruiz, Moreno y Vera (2015) realizaron una investigación con el objetivo de comprobar la importancia del soporte de autonomía, los mediadores psicológicos y la motivación auto determinada sobre la satisfacción docente concluyendo que el soporte de autonomía, los mediadores psicológicos y la motivación académica auto determinada predijeron positivamente la satisfacción docente. El poder satisfacer sus necesidades 
psicológicas básicas resultó ser el mejor indicador para reducir el agotamiento y favorecer la energía necesaria en el desempeño de la labor docente, mientras que los tipos de motivación más autodeterminados se relacionaron negativamente con el agotamiento de los docentes y positivamente con la orientación al trabajo. Los mediadores psicológicos contribuyeron positivamente a la satisfacción docente.

Arias y Velarde (2013) realizaron un trabajo de investigación con el objetivo de comparar los niveles de satisfacción laboral entre dos tiendas por departamento, en Arequipa. Una tienda era peruana y la otra chilena, demostrándose la existencia de niveles de satisfacción más altos entre los empleados de la empresa chilena, sobre todo en cuanto al salario recibido, la organización del trabajo, las posibilidades de ascenso y la satisfacción con la empresa. En la compañía peruana se encontraron relaciones bajas, pero significativas entre el sexo del trabajador y su salario; mientras que en la empresa extranjera, las relaciones más fuertes se dieron entre el salario y la organización del trabajo, la edad y la satisfacción con los subordinados y superiores.

\section{Satisfacción laboral}

De acuerdo con Gonzales, Sánchez y López-Guzmán (2011) la satisfacción laboral es una actitud global hacia el trabajo cuyas causas son multidimensionales. Con relación al concepto de satisfacción laboral, aplicado al contexto de las organizaciones productivas, Hellriegel y Slocum (2009) afirmaron que la satisfacción laboral es un conjunto de actitudes que un individuo adopta ante diversos aspectos de su trabajo, está vinculada con una baja rotación en el trabajo y representa una actitud general de mucho interés para quienes dirigen equipos de trabajo. Por su parte; Luthans, Avolio y Norman (2007) concluyeron que la satisfacción laboral expresa el nivel en el cual las personas encuentran satisfacción en su empleo. De acuerdo con Hackman y Oldman (1980), los factores que miden la satisfacción laboral son: (a) la satisfacción con el sueldo, (b) la seguridad; (c) lo social; (d) la supervisión y (e) el crecimiento. Además, "La satisfacción laboral hace referencia a la actitud general de una persona hacia su trabajo. Una persona con un alto nivel de satisfacción laboral tiene una actitud positiva hacia su trabajo. Una persona insatisfecha tiene una actitud negativa" (Robbins \& Coulter, 2010, p. 285).

Herzberg (1959, citado en Robbins y Coulter, 2010) afirmó la existencia de factores intrínsecos (motivacionales), relacionados con la satisfacción laboral y de factores extrínsecos (higiénicos), relacionados con la insatisfacción laboral. Consideró que los factores que conllevan a la satisfacción laboral son diferentes a aquellos que llevan a la insatisfacción laboral. Por lo tanto, al eliminar los factores que generan insatisfacción 
laboral se puede evitar que las personas estén insatisfechas, pero no necesariamente satisfechas. Para lograr que los trabajadores se encuentren satisfechos, Herzberg planteo utilizar los factores intrínsecos vinculados con el trabajo mismo.

Hegney, Plank y Parker (2006) indicaron que la satisfacción laboral en el área laboral se encuentra fuertemente determinada por la relación entre el personal y las características del ambiente de trabajo. Landy y Conte (2005) afirmaron que la satisfacción laboral es una actitud positiva o estado emocional que resulta de la valoración del trabajo o de la experiencia laboral. En tanto, Ivancevich \& Donnelly (1968) afirmaron que la satisfacción laboral lleva a un concepto que conduce a su categorización en dos perspectivas diferentes: (a) como un estado emocional, un sentimiento, una actitud o una respuesta afectiva hacia el trabajo; y (b) como el resultado de una comparación o ajuste entre anhelos, necesidades o resultados actuales del puesto y las prestaciones que el empleo ofrece realmente.

\section{Autonomía}

De acuerdo con el Departamento de Educación, Universidades e Investigación del Gobierno Vasco (2010), la autonomía se define como:

Una de las cualidades humanas que nos ofrece la posibilidad de actuar por nosotros mismos y que, por tanto, convierte al ser humano en protagonista irrepetible de su vida. Esta competencia supone "ser capaz de imaginar, emprender, desarrollar y evaluar acciones o proyectos individuales o colectivos con creatividad, confianza, responsabilidad y sentido crítico". Está, por tanto, plenamente vinculada a la formación integral de la persona, como lo están el resto de las competencias básicas (p. 3).

Según el filósofo Kant (citado en Torre de Babel Ediciones, 2016), una persona es autónoma cuando "es capaz de ponerse a sí misma sus propias normas; cuando no se rige por lo que le dicen, sino por un tipo de normas que cree que debería cumplir cualquier persona, le apetezca cumplirlas o no" (párr. 5).

\section{Autonomía del trabajo}

Según Smith (2016), la autonomía es la capacidad de los empleados para el control de su situación laboral. En función del tipo de institución y del sector industrial, la autonomía del empleado podría implicar una opción en la selección de proyectos, funciones o clientes. Asimismo, Smith (2016) y Bharthapudi (2016) afirmaron: los beneficios de una empresa cuando sus empelados tiene autonomía y los tipos de autonomía que influyen 
positivamente en la satisfacción de los trabajadores: el método de trabajo, los horarios y los criterios.

La European Foundation for the Improvement of Living and Working Conditions (2007) elaboró un estudio para medir la satisfacción en el trabajo y resultó que el grado de autonomía personal percibido es con frecuencia el factor de pronóstico más significativo y positivo de la satisfacción laboral. Concluyó que la autonomía conceptual ayuda a mejorar la satisfacción laboral en cuatro aspectos: (a) el compromiso, (b) la participación, (c) el rendimiento y (d) la motivación en el lugar de trabajo. Hackman y Oldham (1976, citado en Robbins y Coulter, 2010) desarrollaron el modelo de las características del puesto, en el cual se encontraron cinco dimensiones básicas del trabajo, sus interrelaciones y su efecto sobre la productividad, motivación y satisfacción de los empleados. Las dimensiones básicas son: (a) variedad de habilidades, (b) identidad de las tareas, (c) importancia de las tareas, (d) retroalimentación y (e) autonomía. Estos autores definieron a la autonomía como el "grado al cual un empleo proporciona considerable libertad, independencia y discreción a un individuo mediante la programación del trabajo y la demarcación de los procedimientos que deben usarse para llevarlo a cabo" (Robbins y Coulter, 2010, p. 349). Por otra parte, de acuerdo con Navarro, Llinares y Montañana (2010), la autonomía es "el grado en que el trabajo permite independencia, libertad y capacidad de decisión para planificar el propio trabajo y elegir los procedimientos con que se ejecuta" (p. 13).

Por tal motivo es importante conocer de cerca este fenómeno en trabajadores de una universidad, donde la diversa condición profesional de los colaboradores y las condicionales laborales donde se desarrollan, nos pueden dar un panorama actual de la educación universitaria en función del personal administrativo que lo integra.

\section{Metodología}

La presente investigación se realizó a través del método hipotético deductivo, basado en un enfoque cuantitativo. Behar (2008) señaló:

En el método hipotético deductivo o de contrastación de hipótesis, se busca determinar la verdad o falsedad de las hipótesis planteadas (que no podemos comprobar directamente, por el carácter de su enunciado general, o ya sean leyes que incluyen términos teóricos), partiendo de la verdad o falsedad de los resultados de las observaciones al fenómeno que origina la hipótesis de análisis, unos enunciados se referirán a objetos y propiedades observables, que se obtienen deduciéndolos de las hipótesis y, cuya verdad o falsedad estamos en condiciones de establecer directamente 
(p. 40).

El tipo de investigación, según el propósito, es aplicada. Esto se fundamenta con Hernández et al. (2010), quien indicó que una investigación aplicada es aquella cuya finalidad es resolver problemas prácticos inmediatos, en orden a transformar las condiciones del acto productivo y mejorar la calidad del producto. Por otra parte, según su alcance, es una investigación correlacional, ya que los estudios correlacionales pretenden responder a preguntas de investigación que existan entre dos o más variables de una muestra y establecer una relación entre ellas.

En el presente estudio, la población estuvo constituida por 179 trabajadores administrativos que laboran en una universidad privada peruana. Para la evaluación se elaboró un Cuestionario, que integran una escala tipo Likert de 1 al 5, donde 1 es totalmente en desacuerdo y 5 totalmente de acuerdo.

Es importante mencionar que para validar los instrumentos se realizó la técnica de juicio de expertos, quienes revisaron la coherencia, claridad, concisión, suficiencia, cumplimiento de objetivos, replicabilidad y relevancia de los ítems presentado en el instrumento de evaluación; en lo referido a la justificación estadística se realizó una prueba piloto buscando obtener información que ayude a incrementar la validez y confiabilidad del instrumento y detectar fallas en la validez de construcción y de contenido.

La administración de los instrumentos fue grupal y bajo la supervisión del equipo investigador. La participación de los trabajadores fue voluntaria y anónima, previo a la administración de los instrumentos se entregó a cada participante una ficha de consentimiento informado, para su respectiva lectura y firma, respetando así los principios éticos. Se expusieron los objetivos de la investigación resaltando que los datos serían analizados de forma anónima y el tratamiento de los datos sería confidencial. La evaluación se realizó en lugares, fechas y horarios acordados entre el equipo de investigadores y la dirección del programa

Con la información obtenida, en primer lugar se realizó un análisis descriptivo de todas las variables, Así como también se analizaron las correlaciones de Pearson entre las variables objeto de estudio. El análisis de datos se realizó con el programa estadístico SPSS ${ }^{\circledR}$ versión 22, estableciéndose un nivel de significancia de .05 (5\%). 


\section{Resultados}

El análisis de los resultados demuestra la correlación directa, moderada y significativa entre la autonomía del trabajo y la satisfacción laboral en trabajadores administrativos de una universidad privada.

\section{Tabla 1}

Coeficiente de correlación de Spearman de las variables: Autonomía del trabajo y satisfacción laboral por género

\begin{tabular}{|c|c|c|c|c|c|c|}
\hline & & Sexo & & & $\begin{array}{l}\text { Autonomía } \\
\text { del trabajo } \\
\text { (agrupado) }\end{array}$ & $\begin{array}{l}\text { Satisfacción } \\
\text { laboral } \\
\text { (agrupado) }\end{array}$ \\
\hline \multirow{12}{*}{$\begin{array}{l}\text { Rho } \\
\text { Spearman }\end{array}$} & \multirow{12}{*}{ de } & \multirow{6}{*}{ Masculino } & $\begin{array}{l}\text { Autonomía del trabajo } \\
\text { (agrupado) }\end{array}$ & $\begin{array}{l}\text { Coeficiente } \\
\text { correlación }\end{array}$ & 1.000 & $.651^{* * *}$ \\
\hline & & & & Sig. (bilateral) & . & ,000 \\
\hline & & & & $\mathrm{N}$ & 74 & 74 \\
\hline & & & $\begin{array}{ll}\text { Satisfacción laboral } \\
\text { (agrupado) }\end{array}$ & $\begin{array}{l}\text { Coeficiente } \\
\text { correlación }\end{array}$ & $.651^{\text {** }}$ & 1.000 \\
\hline & & & & Sig. (bilateral) & ,000 & . \\
\hline & & & & $\mathrm{N}$ & 74 & 74 \\
\hline & & \multirow{6}{*}{ Femenino } & $\begin{array}{l}\text { Autonomía del trabajo } \\
\text { (agrupado) }\end{array}$ & $\begin{array}{l}\text { Coeficiente } \\
\text { correlación }\end{array}$ & 1.000 & $.626^{* *}$ \\
\hline & & & & Sig. (bilateral) & . & .000 \\
\hline & & & & $\mathrm{N}$ & 48 & 48 \\
\hline & & & $\begin{array}{l}\text { Satisfacción } \\
\text { (agrupado) }\end{array}$ & $\begin{array}{l}\text { Coeficiente } \\
\text { correlación }\end{array}$ & $.626^{* *}$ & 1.000 \\
\hline & & & & Sig. (bilateral) & .000 & . \\
\hline & & & & $\mathrm{N}$ & 48 & 48 \\
\hline
\end{tabular}

***. La correlación es significativa en el nivel 0.01 (2 colas).

De los valores obtenidos se confirma que tanto los colaboradores varones como mujeres como tienen un moderado grado de método del trabajo, horarios de trabajo y criterios de trabajo; por lo que se puede afirmar que, a mayor autonomía en el trabajo, mayor satisfacción laboral. 


\section{Tabla 2}

Coeficiente de correlación de Spearman de las variables: método del trabajo y satisfacción laboral

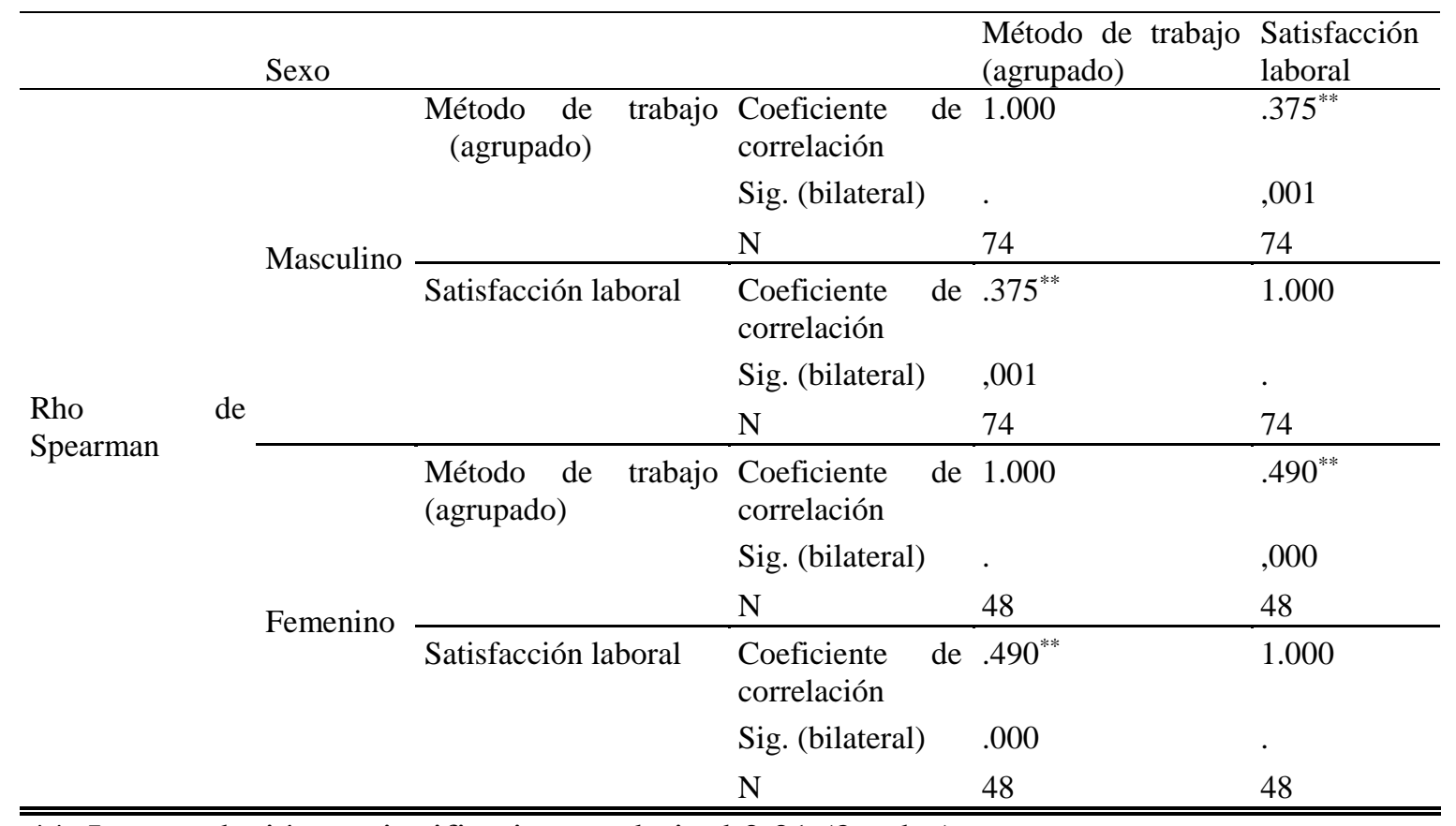

**. La correlación es significativa en el nivel 0.01 ( 2 colas).

De los valores obtenidos, se confirma que los colaboradores perciben que tienen un bajo grado de libertad para planificar el trabajo, para desarrollar el trabajo y un bajo nivel de control en la programación del trabajo. En tanto que las colaboradoras perciben que existe un grado moderado de libertad para planificar el trabajo, para desarrollar el trabajo y un nivel moderado de control en la programación del trabajo. Entonces, se puede afirmar que, a mayor autonomía en el método del trabajo, mayor satisfacción laboral.

\section{Tabla 3}

Coeficiente de correlación de Spearman de las variables: horarios de trabajo y satisfacción laboral

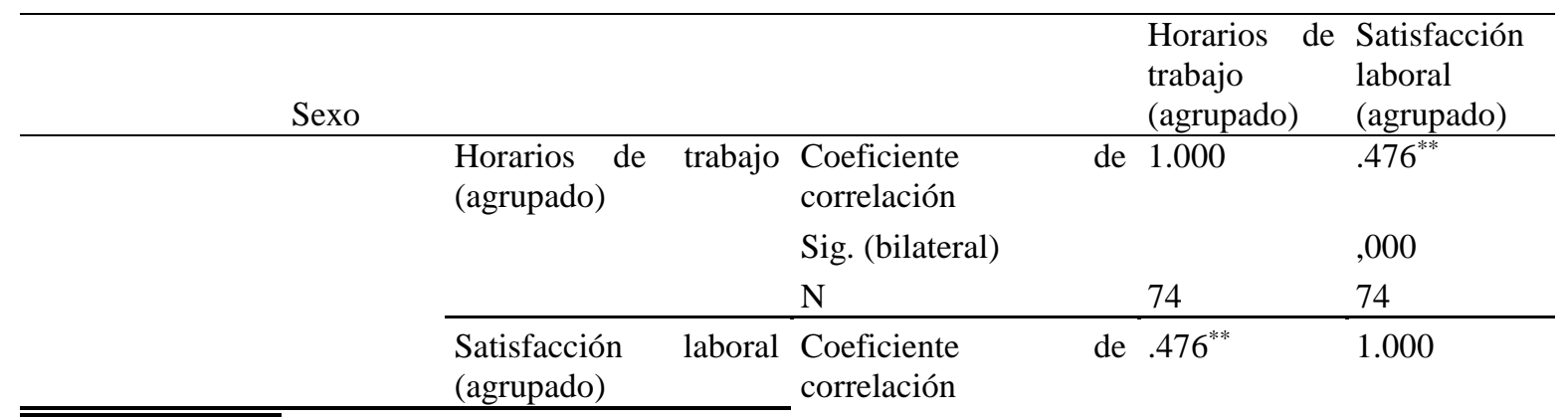




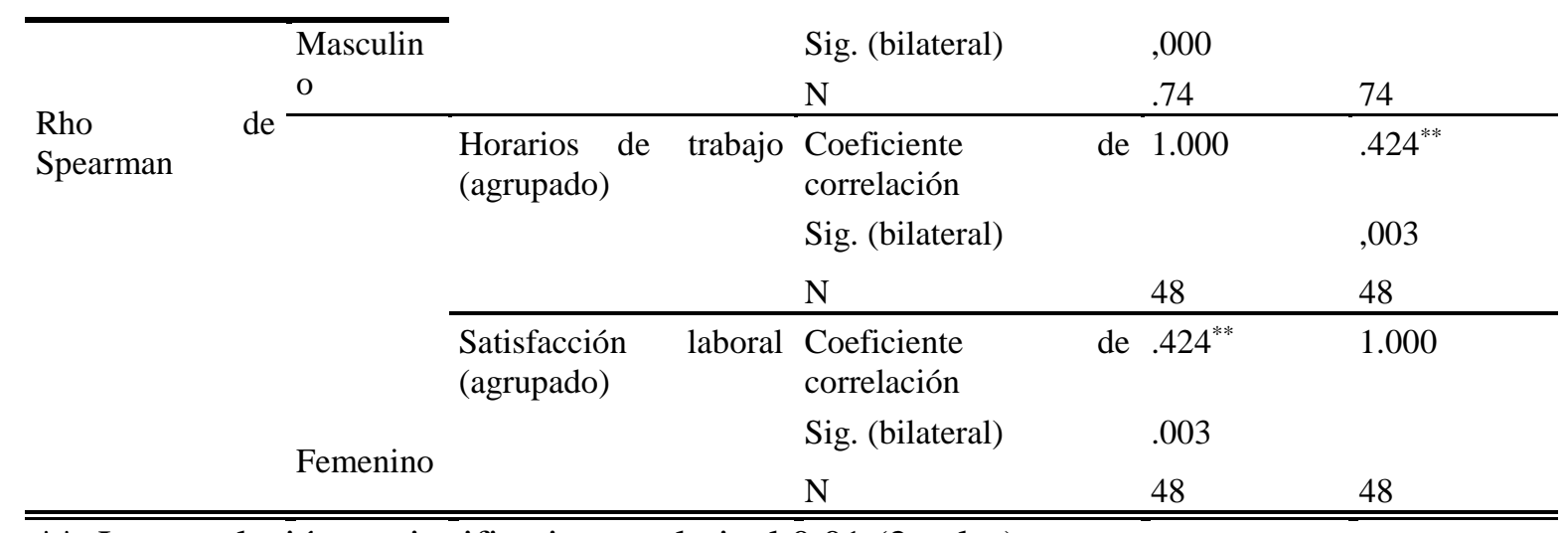

***. La correlación es significativa en el nivel 0.01 (2 colas).

De los valores obtenidos, se confirma que tanto los colaboradores varones como mujeres tienen un moderado grado de nivel de control en el calendario de las actividades del trabajo y puede elegir los procedimientos para ejecutar el trabajo. Al respecto, se puede afirmar que a mayor autonomía en los horarios de trabajo, mayor satisfacción laboral.

\section{Tabla 4}

Coeficiente de correlación de Spearman de las variables: criterios de trabajo y satisfacción labora.

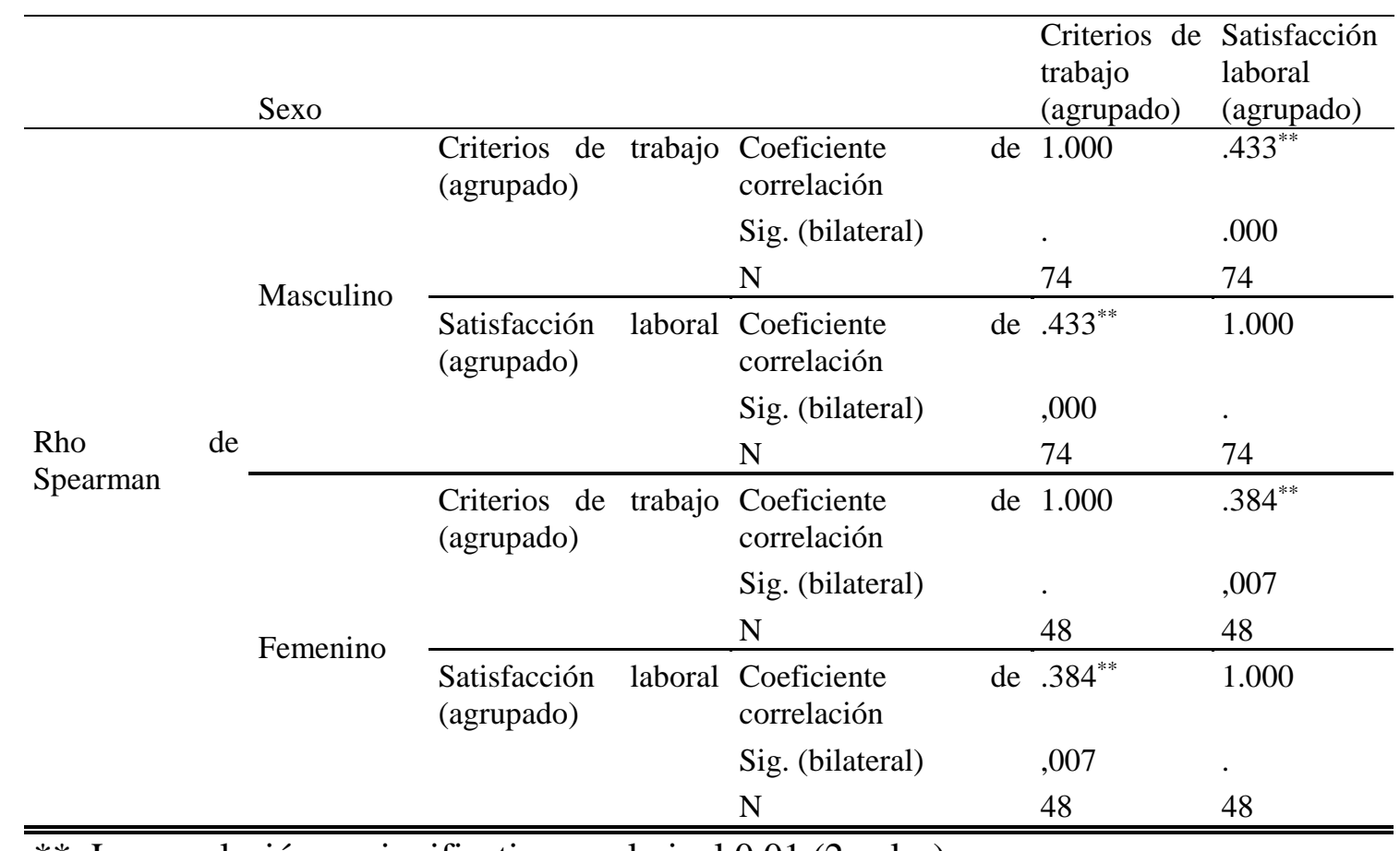

***. La correlación es significativa en el nivel 0,01 (2 colas). 
De los valores obtenidos, se confirma que los colaboradores perciben que tienen un moderado grado de libertad para modificar las normas utilizadas para evaluar el desempeño. Por ello, podemos afirmar que, a mayor autonomía en los criterios de trabajo, mayor satisfacción laboral.

\section{Discusión}

El objetivo del estudio fue encontrar la relación entre la autonomía del trabajo y la satisfacción laboral de los trabajadores administrativos de una universidad, a través del coeficiente de correlación de Pearson encontrando que la correlación es moderada, es decir; tanto los colaboradores varones como mujeres tienen un moderado grado de método del trabajo, horarios de trabajo y criterios de trabajo. Por lo que se puede afirmar que cuando la autonomía del trabajo es real, el nivel de satisfacción laboral es alto, en ambos sexos. De acuerdo al sustento teórico y empírico y los antecedentes revisados, esta relación se explica considerando que el clima organizacional, al tener sustento en elementos tales como calidad de la supervisión, condiciones laborales y comunicación, tiende a influir (casi de manera inevitable), en la motivación y satisfacción laboral de los empleados (Reinoso \& Araneda, 2007).

Además, existe relación directa entre método del trabajo con la satisfacción laboral de los trabajadores administrativos, siendo esta correlación baja en los varones y moderada en las mujeres entre las variables. Se confirma que los colaboradores varones perciben que tienen un bajo grado de libertad para planificar el trabajo, para desarrollar el trabajo y un bajo nivel de control en la programación del trabajo; mientras que las colaboradoras mujeres perciben que existe un grado moderado de libertad para planificar el trabajo, para desarrollar el trabajo y un nivel moderado de control en la programación del trabajo. Se puede afirmar que a menor autonomía en el método del trabajo, menor satisfacción laboral en los colaboradores varones y que a moderada autonomía en el método del trabajo, moderada satisfacción laboral en las colaboradoras mujeres. Estos datos se asemejan a lo encontrado por Oller, Chavero Correo y Ortega, 2015, quienes mencionan que los trabajadores de una empresa perciben un alto nivel de autonomía laboral y, por lo tanto, se encuentran satisfechos con su situación. A pesar de ello, la mayor parte reconoce presiones e influencias procedentes del contexto que limitan o determinan su libertad de expresión.

Por otro lado, existe relación directa entre los horarios del trabajo con la satisfacción laboral de los trabajadores administrativos, siendo ésta una correlación 
moderada entre las variables. Además, se confirma que tanto los colaboradores varones y mujeres tienen un moderado grado de nivel de control en el calendario de las actividades del trabajo y pueden elegir los procedimientos para ejecutar el trabajo. Se puede afirmar que cuando el horario del trabajo se cumple y no hay obligaciones de quedarse fuera de la jornada establecida, el nivel de satisfacción laboral es alta.

Por último, existe relación directa entre autonomía del trabajo con la satisfacción laboral de los trabajadores, siendo esta una correlación directa y moderada en el grupo de varones y baja en el grupo de mujeres entre las variables. Es decir, los colaboradores varones perciben que tienen un moderado grado de libertad para cambiar las normas utilizadas para evaluar el desempeño; mientras que las colaboradoras mujeres perciben que existe un grado bajo de libertad para cambiar las normas utilizadas para evaluar el desempeño. Guillén (2015), nos habla del compromiso organizacional y de su evaluación como parte de los objetivos personales, interpersonales, familiares y colaborativos en comparación al compromiso generado de una socialización en la empresa.

Debido a las limitaciones de tiempo y de recursos, este trabajo se considera una buena guía alternativa para subsecuentes estudios, y adecuada la utilización del enfoque cualitativo, pues así se extrae de manera más fiable la perspectiva del trabajador, quien al fin de cuentas es el objeto de este tipo de estudios.

\section{Referencias}

Arellano Marketing (2013). ¿Qué buscan los jóvenes profesionales peruanos en el mercado laboral? Recuperado de http://www.arellanomarketing.com/inicio/quebuscan-los-jovenes-profesionales-peruanos-en-el-mercado-laboral/

Arias, W., \& Velarde, O. (2013). Job satisfaction among employees from two department stores: a comparative study. Ciencia y trabajo, 15(47), 41-46. Recuperado de Doi: http://dx.doi.org/10.4067/S0718-24492013000200002

Bharthapudi, K. (2016). The Effects of Autonomy on Job Satisfaction. Recuperado de http://www.ehow.com/info_12107936_effects-autonomy-job-satisfaction.html

Behar, D. (2008). Introducción a la Metodología de la Investigación, recuperado de: http://rdigital.unicv.edu.cv/bitstream/123456789/106/3/Libro\%20metodologia \%20investigacion\%20este.pdf

Bonilla, D. M. J., \& Bonilla, E. J. (2016). Clima laboral y su incidencia en la satisfacción de los trabajadores de una empresa de consumo masivo. Ciencia Unemi, 9(18), 26-34.

de: http://ojs.unemi.edu.ec/index.php/cienciaunemi/article/view/301 
Eurofound and EU-OSHA (2014), Psychosocial risks in Europe: Prevalence and strategies for prevention. Publications Office of the European Union: Luxembourg.

European Foundation for the Improvement of Living and Working Conditions (2007).

Measuring job satisfaction in surveys - Comparative analytical report. Tomado de http://www.eurofound.europa.eu/sites/default/files/ef_files/ewco/reports/TN0608T R01/TN0608TR01.pdf

Gobierno Vasco (2010). Competencia para la autonomía y la iniciativa personal. Marco teórico. Recuperado de: http://ediagnostikoak.net/edweb/cas/materialesinformativos/ED_marko_teorikoak/Autonomia_e_iniciativa_personal.pdf

Gómez, S. M. M., \& Barboza, G. A. (2015). Interrelación de la cultura, flexibilidad laboral, alineación estratégica, innovación y rendimiento empresarial. Contaduría y administración, 60(4), 735-756. Doi: https://doi.org/10.1016/j.cya.2014.08.001

Guillén, J. C. (2015). Compromiso laboral del trabajo social en el sector salud. Contaduría y administración, 60(1), 31-51. Doi: https://doi.org/10.1016/S0186-1042(15)72146-7

Hackman, J. R. y Oldman, G. R. (1980). Work Redesign. New Jersey, United States: Prentice Hall Organizational Development Series.

Hegney, D., Plank, A. y Parker, V. (2006). Extrinsic and intrinsic work values: Their impact on job satisfaction in nursing. Journal of Nursing Management, 14 (4), 271-281.

Hellriegel, D.; Slocum, Jr, J. M. (2009). Comportamiento Organizacional (12a. ed.). México, D.F. México: Cengage Learning.

Hernández, R., Fernández, C. y Baptista, M. (2010). Metodología de la Investigación (5ta ed.). México D.F., México: McGraw-Hill.

INEI (2006). Glosario básico de términos estadísticos. Recuperado de: https://www.inei.gob.pe/media/MenuRecursivo/publicaciones_digitales/Est/Lib090 0/Libro.pdf

Ivancevich, J. M. y Donnelly, J. H. (1968). Job satisfaction research: a manageable guide for practitioners. Personnel Journal, 47, 172-177

Landy, F.J. y Conte, J.M. (2005). Psicología industrial: Introducción a la psicología industrial y organizacional. México: McGraw-Hill.

Luceño, L., Martí, J., Rubio, S., y Jaén, M. (2008). Psicología y riesgos laborales emergentes, los riesgos psicosociales. EduPsykhé. Revista de psicología y educación. 7 (2), 111-129

Luthans, F.; Avolio, B.; Avey, J. y Norman, S. M. (2007). Positive Psychological Capital: Measurement and Relationship with Performance and Satisfaction. Personnel Psychology, 60, 541-572. 
Mirás, S., Villoria, E., Álvarez, J., Cotobal, F., \& González, L. (2017). ¿ Cuál es la satisfacción laboral de los trabajadores que solicitan valoración de puesto de trabajo por motivo de salud?. Revista Enfermería del Trabajo, 7(3), 58-64. Recuperado de: https://dialnet.unirioja.es/servlet/articulo?codigo $=6112223$

Moreno, R. R., Alamilla, M. Á. V., Jiménez, I. F., Jiménez, R. F., Mejía, Z. R., Juárez, F. M., \& Pérez, M. G. (2015). Factores explicativos del nivel de satisfacción laboral de los trabajadores de los Institutos de Ciencias Básicas e Ingeniería y Ciencias de la Salud de la Universidad Autónoma del Estado de Hidalgo, una evidencia empírica. XIKUA Boletín Científico de la Escuela Superior de Tlahuelilpan, 3(5). Recuperado de: https://www.uaeh.edu.mx/scige/boletin/tlahuelilpan/n5/e6.html

Navarro, E; Llinares, C; y Montañana, A. (2010). Factores de satisfacción laboral evocados por los profesionales de la construcción en la comunidad valenciana. Revista de construcción, 9, 1, 4-16.

Oller, M., Chavero Correo, P., \& Ortega, E. (2015). La percepción de los niveles de autonomía profesional de los periodistas de Ecuador. Anuario electrónico de estudios en Comunicación Social "Disertaciones", 9(1), 61-83. Doi: http://dx.doi.org/10.12804/disertaciones.09.01.2016.04

Robbins S. P. y Coulter M. (2010). Administración (10a. ed.). Naucalpan de Juárez. México: Pearson educación.

Ruiz, M.; Moreno, J. y Vera J. (2015). Del soporte de autonomía y la motivación auto determinada a la satisfacción docente. European Journal of Education and Psychology, 8, 68-75. Doi: http://dx.doi.org/10.1016/j.ejeps.2015.09.002

Schein, E. (1990). Career anchors: discovering your real values. San Diego, CA: Pfeiffer \& Company.

Smith, E. (2016). What Is Employee Autonomy? Houston Chronicle. Recuperado de http://smallbusiness.chron.com/employee-autonomy-20930.html? hstc

Solórzano, J. V. S., Macias, C. B., Alfonso, Y. R., Betancourt, F. D. R., Apolinario, A. I. L., \& Abreu, J. M. (2018). Incidencia de la insatisfacción laboral en la eficiencia de la gestión. Caso Clínica Estomatológica. Revista Médica Electrónica, 40(3). Recuperado de: http://revmedicaelectronica.sld.cu/index.php/rme/article/view/2755/html_474

Torre de Babel Ediciones (2016). Filosofía medieval y moderna. Kant. Recuperado de http://www.e-torredebabel.com/Historia-de-lafilosofia/Filosofiamedievalymoderna/Kant/Kant-AutonomiaVoluntad.htm 